Material Properties

\title{
Preparation and characterization of functionalized graphene oxide/carbon fiber/epoxy nanocomposites
}

\author{
Alireza Ashori *, Hossein Rahmani, Reza Bahrami \\ Department of Chemical Technologies, Iranian Research Organization for Science and \\ Technology (IROST), Tehran, Iran
}

* Corresponding author. Tel.: +98 2156 275192; Fax: +98 2156275191.

E-mail address: ashori@irost.ir (A. Ashori). 


\section{Abstract}

Graphene oxide (GO) was functionalized using three different diamines, namely ethylenediamine (EDA), 4,4'-diaminodiphenyl sulfone (DDS) and p-phenylenediamine (PPD) to reinforce an epoxy adhesive, with the aim of improving the bonding strength of carbon fiber/epoxy composite. The chemical structure of the functionalized GO (FGO) nanosheets was characterized by elemental analysis, FT-IR and XRD. Hand layup, as a simple method, was applied for 3-ply composite fabrication. In the sample preparation, the fiber-to-resin ratio of 40:60 (w:w) and fiber orientations of $0^{\circ}, 90^{\circ}$, and $0^{\circ}$ were used. The GO and FGO nanoparticles were first dispersed in the epoxy resin, and then the GO and FGO reinforced epoxy (GO- or FGO-epoxy) were directly introduced into the carbon fiber layers to improve the mechanical properties. The GO and FGO contents varied in the range of 0.1-0.5 wt $\%$. Results showed that the mechanical properties, in terms of tensile and flexural properties, were mainly dependent on the type of GO functionalization followed by the percentage of modified GO. As a result, both the tensile and flexural strengths are effectively enhanced by the FGOs addition. The tensile and flexural moduli are also increased by the FGO filling in the epoxy resin due to the excellent elastic modulus of FGO. The optimal FGO content for effectively improving the overall composite mechanical performance was found to be $0.3 \mathrm{wt} \%$. Scanning electron microscopy (SEM) revealed that the failure mechanism of carbon fibers pulled out from the epoxy matrix contributed to the enhancement of the mechanical performance of the epoxy. These results show that diamine FGOs can strengthen the interfacial bonding between the carbon fibers and the epoxy adhesive.

Keywords: Graphene oxide; Carbon fiber; Chemical modification; Mechanical properties 


\section{Introduction}

Carbon fiber/epoxy (CF/EP) nanocomposites with their favorable strength-toweight and stiffness-to-weight ratios are replacing traditional metallic materials in a wide range of applications, such as aeronautical and astronautical structures (e.g., aircraft, space shuttle and satellite) [1-3], and ground vehicles and sports utilities [4, 5]. The mechanical properties of $\mathrm{CF} / \mathrm{EP}$ nanocomposites critically depend on the fibermatrix interfacial adhesion, fiber amount, aspect ratio, orientation with respect to loading direction, etc [6]. Among these, fiber-matrix interfacial adhesion is extremely important since CFs have chemical inertness and poor wettability with most of the polymeric matrices [7]. Good interfacial properties are essential to ensure the efficient load transfer from matrix to fillers, which help reduce stress concentrations and improve overall mechanical properties [8]. Hence, it is necessary to improve the fibermatrix adhesion to enhance mechanical properties.

Many methods have been developed for improving the CF-polymer matrix interfacial adhesion, including electrochemical, chemical, thermal, grafting, coating (sizing) and discharge plasma treatments, etc [9, 10]. Graphene oxide (GO) as a potential multifunctional sizing agent can be uniformly dispersed and firmly adsorbed on the surface of $\mathrm{CFs}$ to prepare a new hierarchical reinforcement. The GO can be produced in large quantities at a relatively low cost and possesses excellent mechanical properties [7]. It has attracted a great deal of scientific interest because of its large surface area $\left(2630 \mathrm{~m}^{2} / \mathrm{g}\right)$, high intrinsic mobility $\left(200,000 \mathrm{~cm}^{2} / \mathrm{Vs}\right)$, high Young's modulus $(\sim 1.0 \mathrm{TPa})$, high thermal conductivity $(\sim 5000 \mathrm{~W} / \mathrm{mK})$, and high optical transmittance $(\sim 97.7 \%)[2,11]$. The GO contains various oxygenated functional groups 
such as epoxide, hydroxyl and carbonyl groups. It is expected that GO coating on CFs will enhance the CF-matrix interfacial adhesion, and thus the applied load can be more effectively transferred from matrix to CFs to enhance composite mechanical properties [12]. However, functionalization of the GO nanosheets is usually required to improve their solubility in organic solvents or compatibility with polymers [13]. The GO is heavily oxygenated, bearing hydroxyl and epoxide functional groups on their basal planes, in addition to carbonyl and carboxyl groups located at the sheet edges $[14,15]$. The presence of these groups makes the functionalization of GO sheets much easier [5, 16]. Among various functional groups, the amine group has a relatively high reactivity and can easily react with many chemicals [17]. Stankovich et al. [18] made the hydrophilic GO nanosheets hydrophobic using isocyanates as modifiers. Niyogi et al. [19] functionalized GO (FGO) with octadecylamine. The GO first reacted with $\mathrm{SOCl}_{2}$ and then the acylated GO was grafted with long-chain aliphatic amine.

Although substantial research has been conducted on improving the interface performance of CF composites, only a small portion has focused on the effect of interface performance on GO/CF/EP nanocomposite $[2,20]$. Therefore, the purpose of the current investigation was to: (1) prepare and characterize FGO using ethylenediamine (EDA), 4,4'-diaminodiphenyl sulfone (DDS), and $p$-phenylenediamine (PPD), (2) fabricate $\mathrm{FGO} / \mathrm{CF} / \mathrm{EP}$ nanocomposites, and (3) determine the role of different functionalization of GO on the mechanical and morphological properties of the nanocomposites.

\section{Materials and methods}




\subsection{Materials}

Graphite powder was used as the starting material for the preparation of graphite oxide (GO). The graphite had a particle size of $\sim 70 \mu \mathrm{m}$, a purity of $99.9 \%$ and a density of $2.25 \mathrm{~g} / \mathrm{cm}^{3}$. Graphite powder, ethylenediamine $\left(\mathrm{C}_{2} \mathrm{H}_{4}\left(\mathrm{NH}_{2}\right)_{2}\right)$, dimethylformamide $\left(\mathrm{C}_{3} \mathrm{H}_{7} \mathrm{NO}\right)$ and thionyl chloride $\left(\mathrm{SOCl}_{2}\right)$ were purchased from Loba Chemie Co. Ltd., India. All other chemicals, such as 4,4'-diaminodiphenyl sulfone $\left(\mathrm{C}_{12} \mathrm{H}_{12} \mathrm{~N}_{2} \mathrm{O}_{2} \mathrm{~S}\right)$, p-nitroaniline $\left(\mathrm{C}_{6} \mathrm{H}_{6} \mathrm{~N}_{2} \mathrm{O}_{2}\right)$, sulfuric acid $\left(\mathrm{H}_{2} \mathrm{SO}_{4}, 98 \%\right)$ and nitric acid $\left(\mathrm{HNO}_{3}, 65 \%\right)$ were obtained from Merck Co. (Germany) and were of reagent grade.

Unidirectional CF used in the research was T700S (with density of $1.8 \mathrm{~g} / \mathrm{cm}^{3}$, average diameter of $7 \mu \mathrm{m}$, and filament number of $12 \mathrm{k}$ ), which was purchased from Toray Industries, Inc (USA).

The matrix system used in this study was a cold-curing bisphenol-A-based epoxy resin Araldit LY 5052 (a novolac epoxy containing 1,4-butanediol diglycidylether) and an aromatic amine hardener HY 5052 (Huntsman Petrochemical Co., USA). Resin-to-hardener ratio of 100:38 by weight was used as recommended by the supplier. Physical properties of the used resin and its hardener are presented in Table 1.

Release agent QZ 13 was a product of Huntsman Petrochemical Co. (USA). It should be noted that the mold was coated with release agent to ensure that the carbon fibers did not adhere to the mold. 


\subsection{Synthesis of $G O$}

The GO was prepared by oxidizing graphite powders based on a modified Staudenmaier method [21]. In brief, $\mathrm{H}_{2} \mathrm{SO}_{4}(18 \mathrm{~mL})$ and $\mathrm{HNO}_{3}(9 \mathrm{~mL})$ were slowly added into a $250-\mathrm{mL}$ round bottom flask and cooled by immersion in an ice bath. Then, raw graphite $(1 \mathrm{~g})$ was added under vigorous stirring. After the graphite powder was well dispersed, $\mathrm{KClO}_{3}(11 \mathrm{~g})$ was added slowly over a $2 \mathrm{~h}$ period to avoid sudden increase in temperature. The oxidation was allowed to proceed for 7 days at room temperature. On completion of the reaction, the mixture was poured into deionized water $(100 \mathrm{~mL})$ and filtered. The graphite oxide was re-dispersed and washed in a 5\% solution of $\mathrm{HCl}$. Then, the product was washed repeatedly with deionized water, until the $\mathrm{pH}$ of the filtrate reached 6 . Subsequently, the GO was obtained by sonicating for 3 $\mathrm{h}$ in a mixture of water and ethanol $(50 \mathrm{~mL})$, and was finally dried in a vacuum desiccator (Fig. 1a).

\subsection{Chemical functionalization of $G O$}

\subsubsection{Functionalization with ethylenediamine (EDA)}

The prepared GO (500 mg) was initially dispersed in thionyl chloride $(30 \mathrm{~mL})$. Then, the mixture was transferred to an oil bath $\left(70{ }^{\circ} \mathrm{C}\right)$ with reflux and stirring for 24 h. The resulting suspension was centrifuged and washed twice with dry tetrahydrofuran (THF) to remove the remaining thionyl chloride. Subsequently, the product (GO-Cl) was dried at $50{ }^{\circ} \mathrm{C}$ for $3 \mathrm{~h}$ in a vacuum desiccator.

The resulting powder was dispersed in EDA $(20 \mathrm{~mL})$ and sonicated for $4 \mathrm{~h}$ at 50 ${ }^{\circ} \mathrm{C}$. Subsequently, the suspension was stirred for $24 \mathrm{~h}$, and then centrifuged and washed 
thrice with THF to make sure that the residual of EDA was thoroughly removed. Finally, the product (EDA-FGO) was dried at $50^{\circ} \mathrm{C}$ for $6 \mathrm{~h}$ in a vacuum oven (Fig. $1 \mathrm{~b}$ ).

\subsubsection{Functionalization with 4,4'-diaminodiphenyl sulfone (DDS)}

The GO-Cl powder was mixed with DDS (2 g) and dispersed in dry dimethyl formamide (DMF, $20 \mathrm{~mL}$ ). After that, the resultant mixture was sonicated for $4 \mathrm{~h}$ at 40 ${ }^{\circ} \mathrm{C}$. Then, the suspension was centrifuged and washed twice with DMF to remove the remaining trace of DDS. Subsequently, it was washed thrice with methanol and dried at $50{ }^{\circ} \mathrm{C}$ overnight in vacuum oven. The final product was obtained by exfoliation of DDS-FGO via ultrasonic homogenizer (Misonix model 4000) at $400 \mathrm{~W}$ for $20 \mathrm{~min}$ (Fig. 1c).

\subsubsection{Functionalization with p-phenylenediamine (PPD)}

The GO-Cl powder was mixed with $p$-nitroaniline (PNA) $(1 \mathrm{~g})$ and then dispersed in dry diethyl ether $(60 \mathrm{~mL})$. The mixture was sonicated in the ultrasonic bath for $3 \mathrm{~h}$ at $35^{\circ} \mathrm{C}$. After sonication, this mixture was stirred for $24 \mathrm{~h}$ and then centrifuged and washed thrice with diethyl ether to ensure the residual of PNA was removed. To reduce nitro groups, PNA-FGO was mixed with stannous chloride $\left(\mathrm{SnCl}_{2}, 2 \mathrm{~g}\right)$ and then dispersed in DMF (30 mL) and refluxed for $18 \mathrm{~h}$. Subsequently, the mixture was centrifuged and washed twice with DMF to remove all the remaining $\mathrm{SnCl}_{2}$. Finally, the powder (PPD-FGO) was dried overnight in a vacuum oven (Fig. 1d).

\subsection{Preparation of composites}


Composites were prepared by a hand lay-up process, using a fiber-to-resin ratio of 40:60 (w:w). Composite panels $\left(20 \times 20 \mathrm{~cm}^{2}\right)$ with thicknesses of $0.40 \pm 0.02 \mathrm{~mm}$ (each ply was approximately $0.13 \mathrm{~mm}$ thick) were fabricated. Before the hand lay-up process, the mold was coated with a release agent to ensure that the fibers would not adhere to the mold. Reinforcement fibers were cut and laid on the mold surface. The fiber orientation (angles) of $0^{\circ}, 90^{\circ}$, and $0^{\circ}$ were used for all the experiments. The GOEP and FGO-EP samples were prepared by mixing and ultrasonic dispersion for 20 and 90 min, respectively. Addition of GO and FGO were based on $0.1,0.3$ and 0.5 wt $\%$, and then the fibers were impregnated evenly with the reinforced resin. The epoxybrushed fibers were carefully stacked up and aligned. The next fabrication process was vacuum bagging. A layer of peel ply was laid down on the specimen because the resin will not stick to peel ply. Then, a layer of breather cloth was laid down to soak up excess resin and ensure an adequate path for the vacuum. Subsequently, the specimen was placed in a plastic vacuum bag and sealed to isolate it from the atmosphere. A vacuum was then applied to the enclosed specimen for $4 \mathrm{~h}$ to reduce the pressure below $3.5 \mathrm{kPa}$. The sample was compacted by the vacuum, and the breather cloth collected the excess resin and removed the air bubbles and volatiles. Also, the pressure creates a uniform surface on the top of the specimen sheet. Finally, the workpieces were placed in an autoclave at $110{ }^{\circ} \mathrm{C}$ for $5 \mathrm{~h}$.

\subsection{Characterization of materials}

X-ray diffraction (XRD) patterns were recorded on a Philips PW1800 diffractometer with $\mathrm{CuK} \alpha$ radiation $(\lambda=1.542 \AA)$ for crystalline phase identification. The sample was scanned from $5^{\circ}$ to $60^{\circ}$. The FT-IR analysis of the nanocomposite was 
conducted in a Philips spectrophotometer, model PU9624, using the transmittance method with wavelength between 400 and $4000 \mathrm{~cm}^{-1}$ at a resolution of $4 \mathrm{~cm}^{-1}$. Elemental analysis was performed using a CHNSO Elemental Analyzer Costech model ECS 4010 apparatus. Morphological structures of samples were studied by scanning electron microscopy (SEM, TESCAN model WEGA-II), operating at an accelerating voltage of $15.0 \mathrm{kV}$.

\subsection{Mechanical testing}

Mechanical testing was performed following ISO 537 standard for tensile properties and ISO 178 for flexural properties. The specimens were cut from the panels parallel to the $\mathrm{x}$-axial direction (based on the first layer). Tensile and bending tests were conducted using a Universal Testing Machine (Instron model 5566), with a constant crosshead speed of $5 \mathrm{~mm} / \mathrm{min}$ at room temperature. An extensometer, with an $80 \mathrm{~mm}$ gauge length, was used to measure the displacement of the specimens. Three replicate tests were conducted for each treatment to obtain a reliable mean and standard deviation.

\section{Results and discussion}

\subsection{Characterization of $G O$ and FGOs}

\subsubsection{XRD}

Fig. 2 shows the XRD patterns of graphite powder and GO. A sharp peak at $2 \theta=26.6^{\circ}$ can be distinguished for graphite, which is assigned to the diffraction of $(002)$ plane of the well-ordered graphene with an interlayer spacing of $0.336 \mathrm{~nm}$ [7]. After oxidation, the diffraction peak of GO shifts to $2 \theta=10.6^{\circ}$, corresponding to an interlayer 
spacing of $0.986 \mathrm{~nm}$. The increase of the interlayer spacing is attributed to the existence of oxygen-containing functional groups on the surface of GO. This means that the graphite was adequately oxidized to GO using the Staudenmaier method.

\subsubsection{FT-IR}

The FTIR spectra of GO and FGOs are shown in Fig. 3. It shows that the GO contains oxygenated functional groups. The characteristic peak of GO at $3430 \mathrm{~cm}^{-1}$ is attributed to the stretching vibration of $\mathrm{OH}$ group. The characteristic peak at $1720 \mathrm{~cm}^{-1}$ corresponds to the stretching vibrations of carbonyl $(\mathrm{C}=\mathrm{O})$ and carboxy $(\mathrm{COOH})$ groups, while the peaks at $1580,1396,1264,1112 \mathrm{~cm}^{-1}$ are attributed to the stretching vibration of aromatic $\mathrm{C}=\mathrm{C}$, carboxyl $\mathrm{C}-\mathrm{O}$, epoxy $\mathrm{C}-\mathrm{O}$, and alkoxy $\mathrm{C}-\mathrm{O}$, respectively. These indicate that the surface of GO contains hydroxyl, carboxyl and epoxide groups, which will enable the GO or FGO to have enhanced interfacial adhesion with polymer matrices $[5,7]$.

Moreover, the FT-IR results show that the oxygenated functional groups of GO were converted to other functional groups through reactions with the above-mentioned diamines (i.e. EDA, DDS, and PPD). The spectra of the FGOs have several new peaks that are not present in the GO spectrum. The peaks appearing at $3500-3700 \mathrm{~cm}^{-1}$ and 1600-1700 $\mathrm{cm}^{-1}$ correspond to amide N-H stretch and amide CO stretch, respectively, which are assigned to the amide functionalization of the GO. The disappearance of the peaks located at 1720,1396 , and $1264 \mathrm{~cm}^{-1}$ in the GO spectrum suggests that the carboxyl and epoxide groups of the GO have reacted with the $\mathrm{NH}_{2}$ groups of the diamines. 


\subsubsection{Elemental analysis}

With the aim of detecting the elemental composition of the FGOs, the elemental analysis was performed. The carbon, hydrogen, nitrogen and sulfur contents are provided in Table 2 . The oxygen element is obtained by a balance, supposing that the remaining element is oxygen. As depicted by Table 2, the atomic ratio (or atom number ratio) of $\mathrm{C} / \mathrm{H} / \mathrm{O}$ in $\mathrm{GO}$ is $3.84 / 3.62 / 3.15$. After functionalization, the atomic ratio of carbon increases to 5-6, and the atomic ratio of oxygen content drops to 1.6-2.0 in FGOs, suggesting that the number of oxygenated functional groups on the GO surface has decreased. In addition, the atomic ratio of nitrogen content reaches approximately 0.13-0.50 in FGOs, confirming the conversion of $-\mathrm{COOH}$ groups into $\mathrm{CONH}$ groups, which is consistent with the FT-IR results. Furthermore, the results of elemental analysis also confirmed that after grafting of DDS on GO, the sulfur content increased moderately.

\subsection{Mechanical properties}

The tensile properties of the samples are shown in Fig. 4 and the standard deviations are represented by error bars. As can be seen, the tensile strength increases initially with increase in the GO and FGOs contents and reaches the maximum at 0.3 wt $\%$ GO content, corresponding to an increase of $22.4 \%$ compared to the untreated ones (control) (Fig. 4a). However, it is clearly illustrated that further addition (more than $0.3 \mathrm{wt} \%$ ) of GO and FGOs cannot considerably improve tensile strength. This could be explained by the agglomeration of nanoparticles [22]. The GO reinforced epoxy composites show the obviously enhanced tensile strength due to the fact that the 
interfacial bonding between CFs and epoxy has been improved by the GO addition. Thus, there will be a better load transfer from the matrix to the CFs, allowing the composite to bear a higher applied load [23]. The enhanced CF-EP interfacial adhesion will be further evidenced later by SEM study.

The tensile modulus exhibits a trend similar to the tensile strength, although more variation is observed in the tensile modulus with different GO and FGOs contents (Fig. 4b). The composite tensile modulus increases constantly with increase in the GO content and reaches the maximum at $0.3 \mathrm{wt} \%$, corresponding to an increase of $800 \%$ compared to the un-treated case. At the higher GO and FGOs contents, the tensile modulus of the composites decreased abruptly. It is well known that the GO has a much higher tensile modulus than the epoxy matrix, thus the increase of the composite tensile modulus with increase in the GO or FGOs content is understandable. In polymer composites, a well-dispersed state of reinforcing fillers ensures a maximized strengthened surface area. This will affect the neighboring polymer chains and, consequently, the properties of the whole composite structure. Hence, the homogeneous dispersion of nano particles in the composite allows efficient load transfer between these phases. SEM study supported the agglomeration of graphene nanoparticles.

The tensile strength increased from $694 \mathrm{MPa}$ for the control sample to 737, 831, 747 and $850 \mathrm{MPa}$ for the GO, EDA-FGO, DDS-FGO and PPD-FGO treated composites with $3 \mathrm{wt} \%$, respectively. The FGO specimens have higher bonding strengths than the control and GO-treated specimens. The PPD-FGO-treated samples showed the largest increase in tensile strength. These results show that the functional 
groups play a significant role in increasing the interaction energy between the carbon fibers and epoxy adhesive. The average tensile modulus of the specimens is summarized in Fig. 4b. It can be seen that the tensile strength of the EDA-FGO and DDS-FGO treated composites is greater than that of the other GO and PPD-FGO treated samples. This indicates that the amine functional group is more active than the other functional groups (alkyl and epoxy) in the epoxy adhesive bonding process. On the other hand, amine functional group acts as epoxy hardener, which enables the formation of better bonding between the epoxy and the CFs.

The flexural strength of the untreated and GO- and FGOs-coated CF/EP composites with various amounts of GO and FGOs is depicted in Figs. 4c and d. Except the composite with $5 \mathrm{wt} \% \mathrm{GO}$, the flexural strength increases initially with increase in the GO content and reaches the maximum at $0.3 \mathrm{wt} \% \mathrm{GO}$ content, corresponding to an increase of $76 \%$ compared to the control. Afterwards, the composite flexural strength decreases with further increase in the GO content. The increase of the composite flexural strength can be attributed to the enhancement of the interfacial adhesion between the CFs and the matrix due to the GO- and FGO-coating on the CFs. The enhanced interfacial adhesion allows better load transfer from the epoxy matrix to the CFs [3], and thereby increases the composite flexural strength. The effect of the GOcoating content on the flexural modulus of the CF/EP composites is shown in Fig.4d. It can be seen that the flexural modulus has an increasing trend with increase in the GO and FGOs contents. The introduction of GO coating leads to a maximum increase of $10 \%$ in the flexural modulus at $0.3 \mathrm{wt} \% \mathrm{GO}$ content compared to the un-treated case. 


\subsection{Morphology}

To understand the role of GO and FGOs addition in enhancing the mechanical properties of the $\mathrm{CF} / \mathrm{EP}$ nanocomposites, the tensile fracture surfaces of the composites were investigated using SEM, as shown in Fig. 5. It can be seen from Fig. 5a that the pulled-out CFs have smooth surface and the gaps between the pulled-out fibers and the epoxy matrix are quite large, indicating that the interfacial adhesion is relatively weak for the untreated (control) composite. In addition, the surface of CF is very clean even although very tiny particles of epoxy resin are remained on the surface.

For the GO and DDS-FGO treated composites, the gaps between the pulled-out CFs and the epoxy matrix become much smaller and a large amount of matrix is attached on the pulled-out CF surfaces as compared with the control sample (Figs. 5b and c), indicating that the $\mathrm{CF} / \mathrm{EP}$ interfacial adhesion is relatively strong for the treated cases. For the composites with EDA- and PPD-FGO (Figs. 5d and e), there are the biggest amounts of epoxy matrix attached on the pulled-out CFs surfaces and there are small gaps between the CFs and the matrix. This indicates that the CF/EP interfacial adhesion is optimal for the $0.3 \mathrm{wt} \%$ case, while a further increase in the GO and FGO contents to $0.5 \mathrm{wt} \%$ will lead to a reduced interfacial adhesion strength but is still much better than for the untreated ones (Fig. 4a). This is possibly attributed to the FGO agglomeration occurring on the $\mathrm{CF}$ surfaces at the $0.5 \mathrm{wt} \% \mathrm{GO}$ content, leading to a reduced interfacial adhesion. Therefore, it is thought that the red arrows in Figs 5f, $g$ and $\mathrm{h}$ indicate the agglomerated $\mathrm{GO}$ and $\mathrm{FGO}$ particles, respectively. These observations in the tensile fracture surfaces of the composites are consistent with the result for the composite tensile strength. 
After the addition of GO or FGO, some particles are shown on the surface of CFs, which were not shown in the control sample. The range of particle size is $30-50$ $\mathrm{nm}$, which is consistent with GO or FGO. As shown in Figs. 5f, g and h, comparison of the bonding surfaces of the treated composites demonstrates that the CF surfaces became relatively rougher after the addition of GO and FGO nano particles. In addition, these images show that the GO and FGOs are well attached on the bonding surfaces.

\section{Conclusions}

In order to enhance the mechanical properties of $\mathrm{CF} / \mathrm{EP}$ composites, $\mathrm{GO}$ and FGOs were used to reinforce epoxy matrix. The conclusions from this study are summarized as follows:

a) The successful oxidation of graphite was evidenced by the results of different characterization methods such as XRD and FT-IR.

b) Decreasing of oxygenated functional groups and the appearance of aminated groups were proved by the FT-IR spectra and the elemental analysis.

c) In general, the effects of addition of the GO and FGOs on the composite mechanical properties were positive. The results showed that the EDA-FGO at a suitable content of $0.3 \mathrm{wt} \%$ can simultaneously enhance the tensile strength and tensile modulus.

d) After the addition of GO and FGO, the CF-EP interface adhesion enhancement is clearly shown by SEM images of the tensile failure surfaces. 
e) Finally, FGO is shown to be a promising modifier to improve the CF-EP interface adhesion for effectively enhancing the mechanical properties of the composites. 


\section{ACKNOWLEDGEMENTS}

This study was financially supported as a research project by grant \# 92036086 from the Iran National Science Foundation (INSF). 


\section{References}

[1] X. Yang, Z. Wang, M. Xu, R. Zhao, X. Liu, Dramatic mechanical and thermal increments of thermoplastic composites by multi-scale synergetic reinforcement: Carbon fiber and graphene nanoplatelet. Mater. Design 44 (2013) 74-80.

[2] L. Chen, H. Jin, Z. Xu, M. Shan, X. Tian, C. Yang, et al., A design of gradient interphase reinforced by silanized graphene oxide and its effect on carbon fiber/epoxy interface. Mater. Chem. Phys. 145 (2014) 186-196.

[3] W. Fan, J. Li, Y. Zheng, Improved thermo-oxidative stability of three-dimensional and four-directional braided carbon fiber/ epoxy hierarchical composites using graphene-reinforced gradient interface layer. Polym. Test. 44 (2015) 177-185.

[4] Q. Chen, L. Zhang, A. Rahman, Z. Zhou, X.-F. Wu, H. Fong, Hybrid multi-scale epoxy composite made of conventional carbon fiber fabrics with interlaminar regions containing electrospun carbon nanofiber mats. Composites Part A 42 (2007) 2036-2042.

[5] D.W. Lee, L. De Los Santos V, J.W. Seo, L.L. Felix, A. Bustamante D, J.M. Cole, et al., The structure of graphite oxide: Investigation of its surface chemical groups. J. Phys. Chem. B 114 (2010) 5723-5728.

[6] D.Q. Vu, M. Gigliotti, M.C. Lafarie-Frenot, Experimental characterization of thermo-oxidation-induced shrinkage and damage in polymer-matrix composites. Composites Part A 43 (2012) 577-586.

[7] F. Li, Y. Liu, C.-B. Qu, H.-M. Xiao, Y. Hua, G.-X. Sui, S.-Y. Fu, Enhanced mechanical properties of short carbon fiber reinforced polyethersulfone composites by graphene oxide coating. Polymer 59 (2015) 155-165. 
[8] M. Chaharmahali, Y. Hamzeh, G. Ebrahimi, A. Ashori, I. Ghasemi, Effects of nano-graphene on the physico-mechanical properties of bagasse/ polypropylene composites. Polym. Bull. 71 (2013) 337-349.

[9] R.J. Sager, P.J. Klein, D.C. Lagoudas, Q. Zhang, J. Liu, L. Dai, J.W. Baur, Effect of carbon nanotubes on the interfacial shear strength of T650 carbon fiber in an epoxy matrix. Compos. Sci. Technol. 69 (2009) 898-904.

[10] L. Mei, X. He, Y. Li, R. Wang, C. Wang, Q. Peng, Grafting carbon nanotubes onto carbon fiber by use of dendrimers. Mater. Lett. 64 (2010) 2505-2508.

[11] A. Ashori, Effects of graphene on the behavior of chitosan and starch nanocomposite films. Polym. Eng. Sci. 45 (2014) 2258-2263.

[12] Z. Zhang, X. Fan, C. Yan, H. Li , Y. Zhu, X. Li, et al., Interfacial microstructure and properties of carbon fiber composites modified with graphene oxide. ACS Appl. Mater. Interf. 4 (2012) 1543-1552.

[13] S. Stankovich, D.A. Dikin, G.H.B. Dommett, K.M. Kohlhaas, E.J. Zimney, E.A. Stach, et al. Graphene-based composite materials. Nature 442 (2006) 282-286.

[14] X.-Z. Tang, W. Li, Z.-Z. Yu, M.A. Rafiee, J. Rafiee, F. Yavari, et al., Enhanced thermal stability in graphene oxide covalently functionalized with 2-amino-4,6didodecylamino-1,3,5-triazine. Carbon 49 (2011) 1258-1265.

[15] J.-L. Yan, G.-J. Chen, J. Cao, W. Yang, B.-H. Xie, M.-B. Yang, Functionalized graphene oxide with ethylenediamine and 1,6-hexanediamine. New Carbon Mater. 27 (2012) 370-376.

[16] D.C. Marcano, D.V. Kosynkin, J.M. Berlin, A. Sinitskii, Z. Sun, A. Slesarev, et al., Improved synthesis of graphene oxide. ACS Nano 4 (2010) 4806-4814. 
[17] Y. Hu, J. Shen, N. Li, M. Shi, H. Ma, B. Yan, et al., Amino-functionalization of graphene sheets and the fabrication of their nanocomposites. Polym. Compos. 31 (2010) 1987-1994.

[18] S. Stankovich, R.D. Piner, S.T. Nguyen, R.S. Ruoff, Synthesis and exfoliation of isocyanate-treated graphene oxide nanoplatelets. Carbon 44 (2006), 3342-3347.

[19] S. Niyogi, E. Bekyarova, M.E. Itkis, J.L. McWilliams, M.A. Hamon, R.C. Haddon, Solution properties of graphite and graphene. J. Amer. Chem. Soc. 128 (2006) 7720-7721.

[20] C.Y. Lee, J.-H. Bae, T.-Y. Kim, S.-H. Chang, S.Y. Kim, Using silanefunctionalized graphene oxides for enhancing the interfacial bonding strength of carbon/epoxy composites. Composites Part A 75 (2015) 11-17.

[21] S. Sheshmani, A. Ashori, M. Arab Fashapoyeh, Wood plastic composite using graphene nanoplatelets. Int. J. Biolog. Macromol. 58 (2013) 1-6.

[22] S.S. Zeng, C. Reyes, J.J. Liu, P.A. Rodgers, S.H. Wentworth, L.Y. Sun, Facile hydroxylation of halloysite nanotubes for epoxy nanocomposite applications. Polymer 55 (2014) 6519-6528.

[23] K. Song, Y. Zhang, M.L. Minus, Polymer interphase self-reinforcement and strengthening mechanisms in low-loaded nanocomposite fibers. Macromol. Chem. Phys. 216 (2015) 1313-1320. 


\section{Tables Captions:}

Table 1. Physical properties of the used resin and hardener

Table 2. Compositions of Go and FGOs calculated by elemental analysis

\section{Figures Captions:}

Fig.1. Structures of the GO and FGOs used in this study

Fig. 2. XRD patterns of graphite and graphene oxide

Fig. 3. FT-IR spectra of GO and FGOs

Fig. 4. Mechanical properties of treated and untreated composites

Fig. 5. SEM images of the composites; (a) control, (b) with $0.3 \mathrm{wt} \% \mathrm{GO}$, (c) with 0.5 wt $\%$ DDS-FGO, (d) with 0.3 wt $\%$ EDA-FGO, (e) with 0.3 wt $\%$ PPD-FGO, (f) bonding surface of composite with $0.5 \mathrm{wt} \%$ GO flakes, with $0.5 \mathrm{wt} \%$ EDA-FGO flakes, and (h) agglomeration of $0.5 \mathrm{wt} \%$ DDS-FGO flakes. 
Table 1. Physical properties of the used resin and hardener

\begin{tabular}{|c|c|c|c|}
\hline Properties & $\begin{array}{c}\text { Araldite }^{\circledR} \mathrm{LY} \\
5052\end{array}$ & $\begin{array}{c}\text { Aradur }^{\circledR} \\
5052\end{array}$ & ISO \\
\hline Appearance & clear liquid & clear liquid & - \\
\hline Color (Gardner) & $\leq 2$ & $\leq 4$ & 4630 \\
\hline Viscosity at $25^{\circ} \mathrm{C}$ & $1000-1500 \mathrm{cps}$ & $40-60 \mathrm{cps}$ & $12058-1$ \\
\hline Density at $25^{\circ} \mathrm{C}$ & $1.17 \mathrm{~g} / \mathrm{cm}^{3}$ & $0.94 \mathrm{~g} / \mathrm{cm}^{3}$ & 1675 \\
\hline Flash point & $\geq 140^{\circ} \mathrm{C}$ & $\geq 110^{\circ} \mathrm{C}$ & 2719 \\
\hline
\end{tabular}


Table 2. Compositions of GO and FGOs calculated by elemental analysis

\begin{tabular}{ll}
\hline & $\mathrm{C} / \mathrm{H} / \mathrm{O} / \mathrm{N} / \mathrm{S}$ (atomic ratio) \\
\hline GO & $3.84 / 3.62 / 3.15$ \\
EDA-FGO & $5.34 / 2.08 / 1.63 / 0.50$ \\
DDS-FGO & $5.04 / 2.16 / 2.14 / 0.13 / 0.04$ \\
PPD-FGO & $5.83 / 2.30 / 1.62 / 0.13$ \\
\hline
\end{tabular}




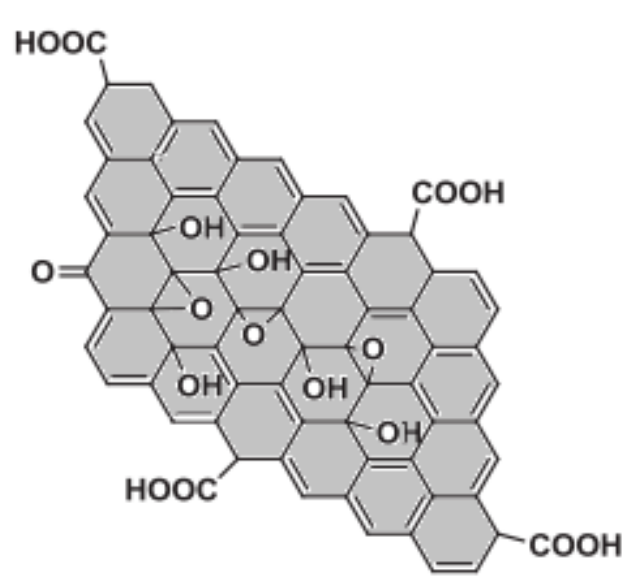

GO

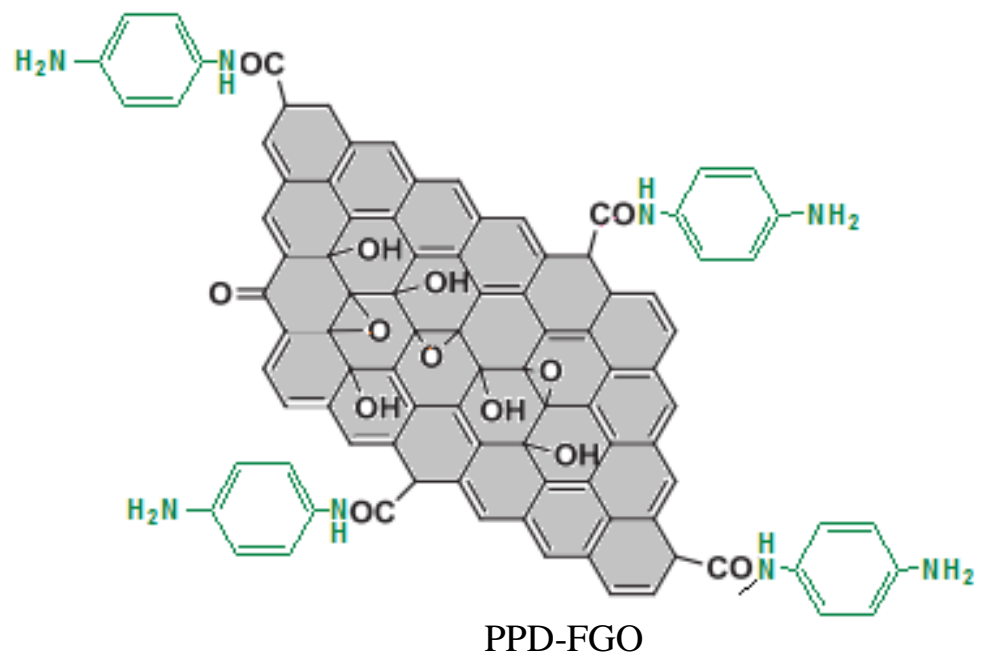

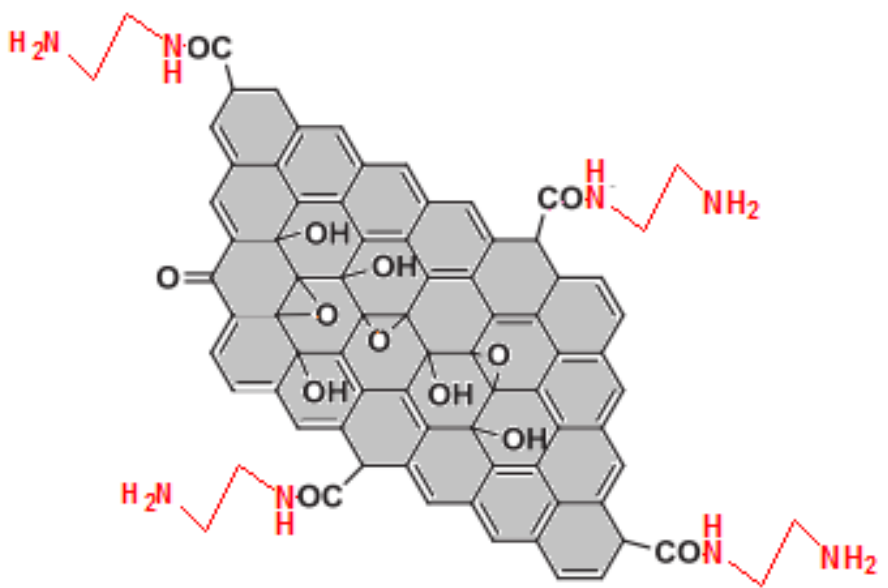

EDA-FGO

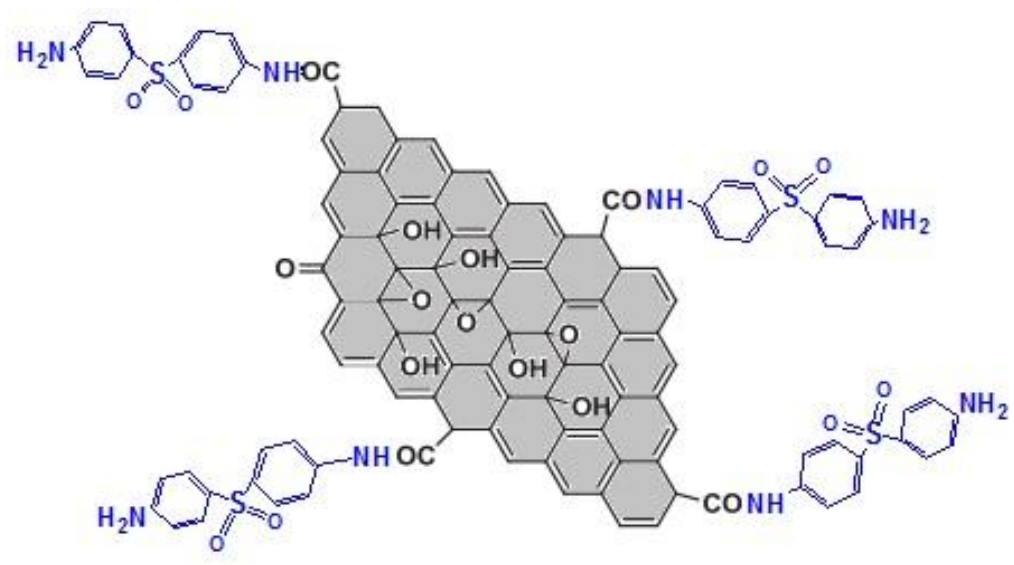

DDS-FGO

Fig.1. Structures of the GO and FGOs used in this study 


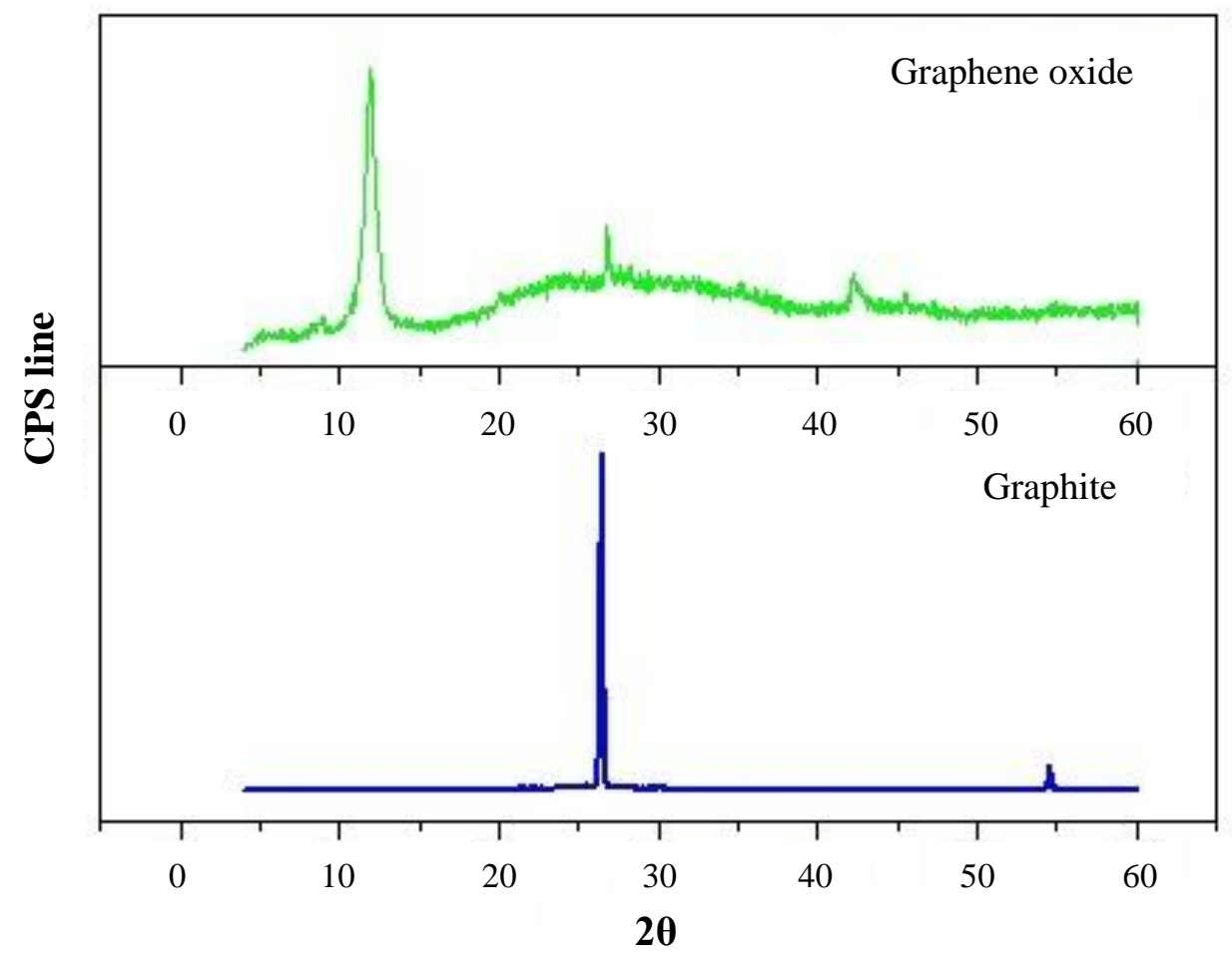

Fig. 2. XRD patterns of graphite and graphene oxide 


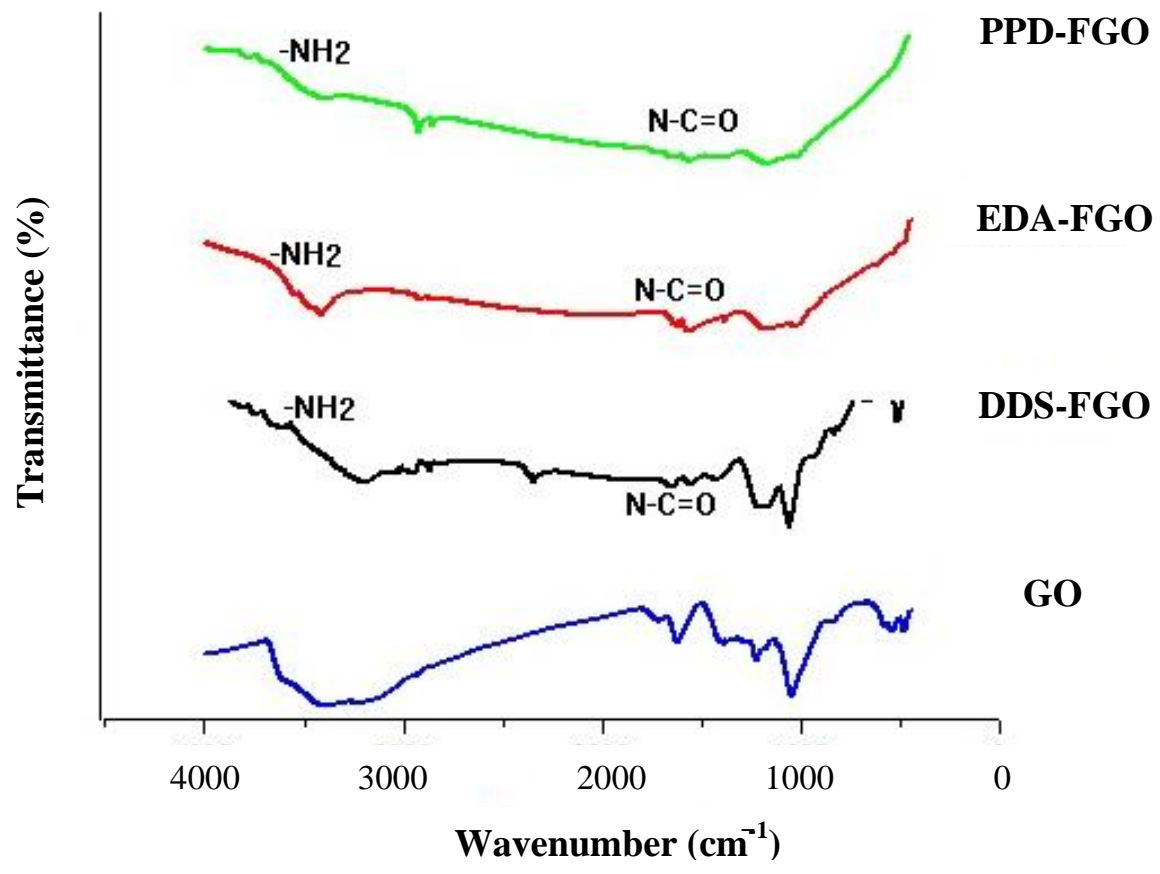

Fig. 3. FT-IR spectra of GO and FGOs 

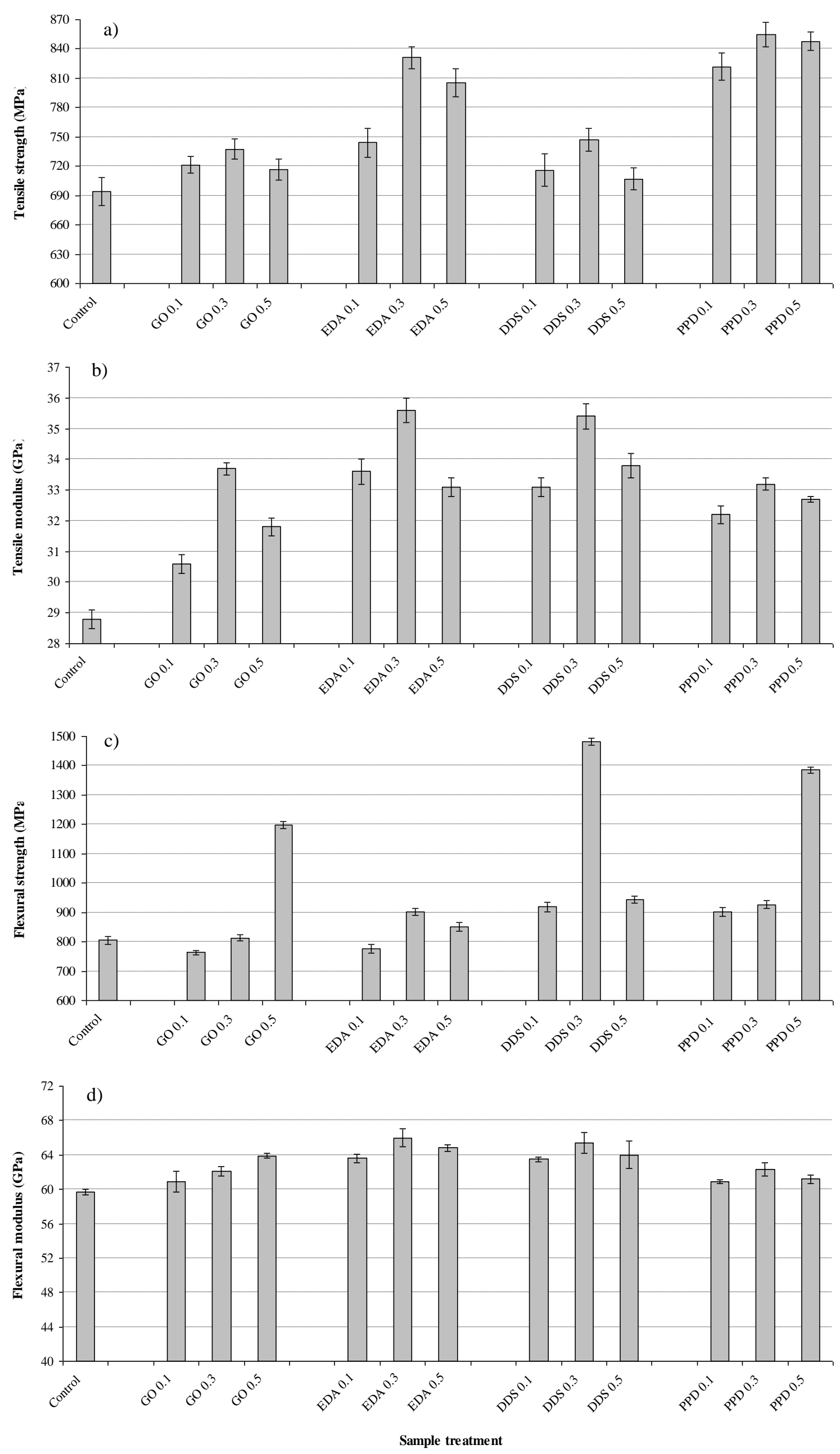

Fig. 4. Mechanical properties of treated and untreated composites 